\title{
LOS SISTEMAS MULTIMEDIA DIGITALES INTERACTIVOS EN LOS MUSEOS
}

Rocío Mihura-López1: Universidade da Coruña. España mihura@udc.es

Viviana Barneche-Naya: Universidade da Coruña. España viviana.barneche@udc.es

Luis A. Hernández-Ibáñez: Universidade da Coruña. España lhernandez@udc.es

\section{RESUMEN}

Los museos basan su potencial educativo en la posibilidad por parte del visitante de vivir una experiencia de forma directa, ya sea a partir de un objeto $\mathrm{u}$ ambiente recreado. Como entidades que dependen directamente de la sociedad y que experimentan a la vez sus avances, han implementado la tecnología multimedia digital en las exposiciones con mayor o menor éxito. Uno de los resultados positivos es su atractivo para todo tipo de público y su potencial para mostrar la complejidad histórica o social de un objeto.

PALABRAS CLAVE: Museo - Interactivo - Digital - Multimedia - Experiencia

\footnotetext{
${ }^{1}$ Autor correspondiente

Rocío Mihura-López: Universidade da Coruña. España

Correo: $\underline{\text { mihura@udc.es }}$
} 


\title{
DIGITAL INTERACTIVE MULTIMEDIA DEVICES IN MUSEUMS
}

\begin{abstract}
Museums base their educational potential on the visitors' chances of living a direct experience, through an object or a recreated ambient. As institutions they are run by the society and evolve at the same time. Therefore museums have implemented digital multimedia technology in their expositions, more or less successfully. One of the positive consequences is the attraction of these devices for all kinds of public and their ability to showing social or historical complexity of an object.
\end{abstract}

KEY WORDS: Museum - Interactive - Digital - Multimedia - Experience

\section{INTRODUCCIÓN}

Las TIC digitales están actualmente presentes en todos los sectores de la sociedad, ya sea en el ámbito laboral o en el sector del ocio. Los museos por su parte, digitalizan sus colecciones para una óptima catalogación, comparten datos on-line y ofrecen información y recursos que mejoran las visitas a través de sus web, empleando la tecnología como una nueva herramienta de comunicación consonante con el público del siglo XXI. Ya han transcurrido más de veinte años desde que un museo presentase su primera web en la que, de forma muy básica, se ofrecía al visitante información sobre horarios, situación y tarifas. Tras años de implantación paulatina de estas herramientas, se produjo durante los años 90 en los museos una confrontación, debido a que, según denunciaban algunos teóricos, los ordenadores pretendían substituir a los objetos expuestos, ya que el uso de estos dispositivos se limitaba principalmente a ofrecer información audiovisual, acompañando o no objetos muebles incluidos en la exposición. A medida que la tecnología fue avanzando, se incrementaron las posibilidades museográficas de estos sistemas, de forma que poco a poco dejaron de competir con el objeto real adquiriendo una entidad como objeto portador de una experiencia para el visitante.

\section{DESARROLLO}

\subsection{Museos, educación y sistemas digitales interactivos}

El museo es una entidad que depende directamente de la sociedad que lo acoge. Este hecho ha de reflejarse directamente en sus objetivos y razón de ser. Según el estudio que Koester realiza en "Interactive Multimedia in American Museums" (Koester, 1993) el fin último de los museos ha ido variando a lo largo de la historia conforme cambian 
los valores de la sociedad que representa, desde el coleccionismo de objetos como símbolo de conquista cultural hasta su papel como exponentes de riqueza o prestigio económico o cultural.

En el siglo XVIII las colecciones comenzaron a hacerse públicas, lo que cambió ligeramente la perspectiva que el museo tenía sobre el visitante. A pesar de esto, el público seguía siendo considerado un elemento ajeno y a veces poco deseable. Con la creación de los grandes museos nacionales europeos se empieza a fomentar la afluencia del público, empleando herramientas expositivas que favorezcan la comprensión y asimilación de contenidos de la muestra (Zetterberg, 1968). En los EEUU los museos se crean con una perspectiva más democrática que en Europa, fomentando la función educativa de esta entidad. La Smithsonian Institution, por ejemplo, nace a mediados del siglo XIX con el propósito de incrementar y difundir el conocimiento.

El éxito social de la Great Exhibition de Londres de 1851 provoca que el hecho de visitar un museo adquiera una importancia política que hasta entonces no había disfrutado; el público por lo tanto, aparece como un valor cuantificable y en ascenso, por lo que había que definir claramente la función del museo en la sociedad y potenciarla de forma competitiva. Además de valores asociados a la supuesta categoría social y cultural que otorgaba el ser visitante asiduo de museos, la función educativa se contempla como una misión claramente establecida.

A comienzos del s. XX, los comisarios y directores de museo detectaron la necesidad de abandonar la actitud de open-education respecto al visitante, con el fin de incrementar la afluencia y fidelidad. Esto repercutiría en el museo con una mayor cantidad de fondos $\mathrm{y}$ donaciones. Los elementos educativos comienzan a entremezclarse con el entretenimiento y la diversión (infotainment, edutainment) bajo la supervisión de profesionales especialistas en el diseño de exposiciones. El visitante medio comienza a sentirse más gratificado tras la visita al museo y siente deseos de repetir la experiencia. También se abre más el ámbito de posibles usuarios, mayor rango de edades y menor necesidad de nivel un alto nivel cultural previo a la visita. En conclusión, el museo se acerca a su público. Este acercamiento a través de la diversión se contempla como algo positivo y constructivo. Museo, educación y entretenimiento son conceptos que comienzan a aparecer unidos y no contrapuestos.

\subsection{El museo como lugar de aprendizaje}

La conocida taxonomía cognitiva de Bloom orientada a procesos educativos, concluye que el aprendizaje puede producirse en cualquiera de tres dominios psicológicos: cognoscitivo, psicomotor o afectivo. Los museos basan su potencial de aprendizaje en el último de estos dominios, potenciando la llamada educación informal en contraposición a la educación formal de las escuelas tradicionales. Aunque el objetivo es el mismo, los museos ofrecen la posibilidad de aprender directamente del objeto $\mathrm{u}$ ambiente, fijando el contenido con ayuda de la experiencia diresta. mientras a11e las esc11elas transmiten información nreviamente filtrada $v$ 
textualizada a través de libros y docentes.

Los investigadores Brown, Screven, Koran y Beer, citados en Bitgood (Bitgood, 1988) observan que, mientras que la educación formal sistematiza y mide la cantidad de conocimientos transmitidos, la educación informal se basa en la exploración, ya que el visitante descubre la información según sus propios intereses. Bitgood (Bitgood, 2002) enumera una serie de áreas en las que el aprendizaje informal difiere del formal, con el fin de lograr gracias a una mejor definición de este proceso el éxito en el diseño de las exposiciones.

Comienza resaltando que la enseñanza informal propone el aprendizaje a partir de la experiencia: En el museo los objetos se captan a partir de estímulos: Los medios de enseñanza del museo son directos, visuales o multisensoriales, experienciales y proporcionan la información en pequeñas dosis. Además, la enseñanza informal no se produce en un aula. En el museo el entorno de aprendizaje es dinámico y desconocido para el visitante, el nivel de interés se mantiene alto aunque la retención de contenidos es más baja.

Como consecuencias derivadas del aprendizaje destaca que el aprendizaje informal no realiza exámenes sobre los conocimientos adquiridos, no hay premio ni amonestación posterior a la visita. En un museo, no suele ser habitual, excepto en las visitas de escolares, que se efectúe ninguna prueba de conocimiento o examen. A pesar de que este autor solo enumera ventajas relativas a este tipo de educación, en realidad propone que ambos sistemas deben coexistir y cooperar para alcanzar objetivos comunes.

\subsection{Educación y tecnología multimedia interactiva en el museo}

Podemos hallar encontrar posiciones encontradas respecto a este binomio. Como experiencia positiva se citará a Gadner y a Hawkey. Gardner (Gardner, 1991 a), tras identificar siete modos en los que el individuo comprende el mundo (lógicomatemático, lingüístico, espacial, musical, cinético, intra-personal e inter-personal), concluye que la tecnología multimedia permite a cada persona reforzar su propio sistema cognitivo, sin imposiciones externas de la mayoría. Esto se produce gracias a la capacidad de esta tecnología para permitir un aprendizaje personalizado, según la demanda, interés y conocimiento previo del usuario.

El argumento propuesto por Gadner, no parece, sin embargo, aprovechar las posibilidades de estos medios en el entorno museo, ya que esta propuesta también coincide con las ventajas de la tecnología multimedia en el hogar. El usuario obtendría beneficios similares delante de un ordenador en su propia casa.

La propuesta de Hawkey es más concreta y específica. Tras investigar los diversos 
modos en que la tecnología digital se emplea en los museos para la educación, determina que las características únicas de la tecnología digital al servicio del aprendizaje encajan con los objetivos del aprendizaje informal activo, y concluye que los museos son un entorno único para establecer esta sinergia (Hawkey, 2002).

Las valoraciones negativas sobre el impacto de las nuevas tecnologías en los museos, parten de la diferencia entre la educación formal (escuela) y la educación informal (museo). Aunque varios estudios avalan las ventajas de la tecnología multimedia en la educación formal impartida en las escuelas, su presencia en el museo no tiene por qué necesariamente resultar igualmente beneficiosa. Como exponente de este criterio, se puede citar a Knell (Knell, 2003). Este investigador enumera una serie de cuestiones negativas relativas a la relación entre museo y tecnología. La primera se refiere al hecho de que las novedades en este tema vienen de la mano de tecnólogos, y no de museólogos (ni de educadores), lo que banaliza el contenido educativo y lo convierte en mera excusa para mostrar un avance tecnológico. En segundo lugar se cuestiona el valor real de la experiencia virtual a la que el individuo puede optar hoy en día en un museo.

\section{CONCLUSIONES}

\section{Ventajas de estos sistemas para los museos}

Tras analizar varias posturas encontradas, se pueden citar como ventajas de estos sistemas para el museo los siguientes puntos:

a) Permiten abarcar la complejidad histórica social de un objeto: acudiendo a Singleton, los documentos multimedia permiten abarcar la complejidad de datos necesarios en esta era de la utilización de la capacidad mediática del objeto en el contexto museológico. La adición entre la información audiovisual, documentales fílmicos sobre la época y entorno de los objetos, hipermedia, simulaciones virtuales, y la interactividad añadida, que capacita al visitante como comisario, y partiendo del propio conocimiento del tema, obtiene resultados personalizados. Todo esto conforma un universo de información impensable sin el empleo de la multimedia.

b) El contenido mostrado no está limitado por el espacio físico del museo: en algunas ocasiones, el espacio disponible para mostrar obras limita la cantidad de las mismas que el museo puede exponer. Los sistemas digitales permiten que el espectador pueda observar infinidad de objetos y compararlos entre sí, evitando problemas de localización geográfica, temporal o tamaño físico.

c) Proporcionan información en la cantidad y profundidad deseada por cada individuo: para el museo a veces resulta difícil colocarse en el lugar del visitante y atraerlo, ya que si el contenido expuesto es demasiado especializado, el rango de espectadores es pequeño, mientras que si ofrece información demasiado banal el visitante no repite la visita. Los interactivos digitales multimedia bueden estructurarse en diferentes 
niveles de complejidad, ofreciendo además información tanto visual, que es normalmente más ligera, como textual, más precisa y profunda. El usuario avanza en la medida que su tiempo e interés requiere.

d) Resultan enormemente atractivos para todas las edades, sobre todo para los más jóvenes.

Los museos contemporáneos compiten a la hora de atraer al público, y no solo entre sí; la variedad de empresas dedicadas al ocio (sea éste o no de índole cultural) crece conforme mejora la sociedad de bienestar. Actualmente asistimos a una transformación de los museos, que buscan la combinación de entretenimiento y aprendizaje como una alternativa competitiva a otras actividades exclusivamente lúdicas (Feber, 1987), (Hooper-Greenhill, et al., 2003). Los estudios sobre los visitantes en los museos, habituales desde los años setenta, coinciden en señalar la necesidad que tienen este tipo de instituciones de conectar con todo tipo de público, de diferentes edades, condición cultural e intereses. Los nuevos medios se han revelado como una herramienta imprescindible para conseguir estos objetivos.

\section{REFERENCIAS}

Bitgood, S. (1988). A comparision of formal and informal learning. Jacksonville: Jacksonville State University.

Bitgood, S. (2002). Environmental psychology in musums, zoos and other exhibition centers. En Handbook of Environmental Psychology. EEUU: Bechtel \& A. Churchman.

Feber, S. (1987). The Boston children's museum. The International Journal of Museum Management and Curatorship.

Gardner, H. (1991). Making schools more like museums. EE.UU: Education Week.

Hawkey, R. (2002). The lifelong learning game: season ticket or free transfer?. Computers E Education, 38.

Hooper-Greenhill, E. (2003). Measuring the outcomes and impact of learning in museums, archives and libraries. Leicester: Research Centre for Museums and Galleries.

Knell, S. (2003). The shape of things to come: museums in the technological landscape. Museum and Society, 1(3): 132-146.

Koester, S. E. (1993). Interactive Multimedia in American Museums. EE.UU: Archives \& Museum Informatics. 
Zetterberg, H. L. (1968). Museums and adult education. London: Evelynm Adams and Mackay.

\section{Rocío Mihura López}

Licenciada en Bellas Artes por la Universidade de Vigo. Máster en Creación y Comunicación Digital por la Universidade da Coruña. PhD candidate, en etapa de Tesis, Universidad de A Coruña. Profesora en la Facultade de Ciencias da Comunicación de la Universidade da Coruña. Sus líneas de investigación abarcan los campos de la creación digital interactiva en el ámbito de la museística y los contenidos audiovisuales dentro de los Museos.

\section{Viviana Barneche Naya}

Arquitecta, Universidad de la República, Uruguay. PhD candidate, en etapa de Tesis, Universidad de A Coruña. Docente de Taller de Anteproyectos, Facultad de Arquitectura, UdelaR (2001-2011). Docente dentro del Máster en Creación y Comunicación Digital, Universidad de A Coruña (2008-2009). Profesora interina, Universidad de A Coruña (2011). Sus líneas de investigación se enmarcan en los campos de la creación digital interactiva, el diseño de ciberarquitectura y contenidos dentro de los Metaversos, la recreación patrimonial, la simulación por computador y las Interfaces Naturales.

\section{Luis Antonio Hernández Ibáñez}

Doctor Arquitecto, Universidad de A Coruña, España. Director del Grupo de Visualización Avanzada en Arquitectura, Ingeniería Civil y Urbanismo - VideaLAB, Director del Máster en Creación y Comunicación Digital. (1998-2009). Profesor Titular en la Escuela Técnica Superior de Ingenieros de Caminos, Canales y Puertos; y de la Facultad de Ciencias de la Comunicación de la Universidad de A Coruña. Autor de múltiples publicaciones en el ámbito de la visualización avanzada, la ciberarquitectura y la recreación del Patrimonio. Sus líneas de investigación en enmarcan en el campo de la creación digital, la simulación por computador, las Interfaces Naturales, los Mundos Virtuales 3D y la Realidad Virtual. Miembro de IEEE, ACM Siggraph, Sigradi y SEAV. 